\title{
EchoGéo
}

47| 2019

Nouvelles géographies de la collecte

\section{À prendre ou à laisser ?}

Ressources, gestes et corps de la récupératrice alimentaire en milieu urbain

Jeanne Guien

\section{OpenEdition}

Journals

Édition électronique

URL : https://journals.openedition.org/echogeo/16521

DOI : 10.4000/echogeo.16521

ISSN : 1963-1197

Éditeur

Pôle de recherche pour l'organisation et la diffusion de l'information géographique (CNRS UMR 8586)

Référence électronique

Jeanne Guien, «À prendre ou à laisser ? », EchoGéo [En ligne], 47 | 2019, mis en ligne le 21 avril 2019, consulté le 10 août 2021. URL : http://journals.openedition.org/echogeo/16521 ; DOI : https://doi.org/ 10.4000/echogeo. 16521

Ce document a été généré automatiquement le 10 août 2021.

EchoGéo est mis à disposition selon les termes de la licence Creative Commons Attribution - Pas d'Utilisation Commerciale - Pas de Modification 4.0 International (CC BY-NC-ND) 


\title{
À prendre ou à laisser?
}

\author{
Ressources, gestes et corps de la récupératrice alimentaire en milieu \\ urbain
}

Jeanne Guien

1 À Paris, qu'elle se fasse solitairement ou dans le cadre de groupes affinitaires, la récupération alimentaire a lieu dans l'espace public de la rue, à des heures fréquentées. Lieu de passages et d'interactions rapides, la rue place la récupération alimentaire dans de véritables situations d'échange, qui questionnent le statut de son objet-limite : le déchet. Objet de rejet pour les uns, ressource pour les autres (Bertolini, 1978), le déchet génère en effet des échanges et des activités variées (Corteel et Le Lay, 2011 ; Benelli $e t$ al., 2017), qui participent de l'activité économique, du « métabolisme urbain » (Barles, 2005), de la constitution socio-démographique des territoires et "écosystèmes" urbains (Bazin et Rullac, 2011), dans un "continuum socio-technique » entre activités formelles et informelles internationalement documenté par le champ très actif des discard studies (Cirelli et Florin, 2015, p. 17).

2 Le déchet génère ainsi toutes sortes de négociations, où s'évaluent la propriété qu'il fut, le bien qu'il sera, l'espace qu'il occupe, les regroupements sociaux que sa gestion engage. En droit appropriable par seule mainmise, le déchet possède une valeur marchande ambiguë (puisqu'on paie pour en être débarrassé par des entreprises qui en tirent profit) qui fait de la récupération un terrain fertile en ces situations de « conflit d'interprétation » qui, selon Florence Weber, se produisent lorsque deux ou plusieurs partenaires d'un échange ne sont pas d'accord « au sujet de la définition même de ce qui circule et dans quel sens » (Weber, 2009, p. 372), révélant la diversité des «cadres matériels et cognitifs » auxquels se réfèrent les acteurs pour légitimer leurs échanges, ou leur refus de l'échange.

3 Cet article se propose de réfléchir aux enjeux économiques et symboliques de la récupération alimentaire en milieu urbain dense (le Nord-Est parisien) et sur une période longue (six années consécutives), à partir d'un témoignage réflexif prenant pour objet des conflits d'interprétation autour de cette pratique. Ce travail s'inscrit dans une démarche de recherche-action (LISRA, 2017), l'auteure étant une récupératrice, une chercheuse et une activiste engagée dans la critique de l'économie 
du gaspillage. Il repose sur une méthode d'autoethnographie (Ellis et al, 2010) : plutôt que de chercher à adopter un point de vue objectif sur une pratique qui ne l'est pas, puisque je recours à la récupération pour mon alimentation quotidienne, je propose de mettre à profit la posture située qui est la mienne pour questionner ma pratique sociologiquement. L'analyse de certaines situations d'échanges montre en effet à quel point les biais de classe, d'âge et surtout de genre sont en jeu dans les interprétations variées des gestes de récupération par les commerçantes ${ }^{1}$, travailleuses du déchet et passants. Le fait d'être ce corps qui se penche, se saisit et se nourrit de déchets alimentaires sans demander le droit à personne fut à l'origine de nombre de ces « épiphanies » qui constituent l'objet de l'autoethnographie (Ellis et al, 2010), révélant dans l'ambiguïté voire le conflit les constructions symboliques des objets, des déchets et des corps humains sur lesquelles reposent l'économie du gaspillage et la répartition genrée des tâches dans une économie marchande.

\section{Le freeganisme à Paris - éléments de contextualisation}

4 Pratiquant la récupération alimentaire à Paris depuis 2012, je ne pourrais qualifier mon activité de «freeganisme » qu'au prix d'une certaine restriction du sens de ce terme. Elle est certes née d'un choix militant et non d'une nécessité économique, dans la volonté de construire des pratiques de boycott du marché dominant, dans un espace urbain où les alternatives, notamment alimentaires, sont peu développées, et où l'idéal d'autonomie matérielle se confronte à d'importantes limites macrotechniques. «Boycott ultime » (Oakes, 2000) (ma traduction), le freeganisme est présenté par ses acteurs historiques comme conclusion d'un parcours militant.

« Après des années passées à essayer de boycotter les produits d'entreprises non éthiques, responsables de violations des droits humains, de destruction écologique et de souffrance animale, beaucoup d'entre nous sont parvenues à la conclusion que, quoi que l'on achète, on finit par encourager un modèle déplorable. Nous avons réalisé que le problème ne vient pas de quelques vilaines entreprises mais du système tout entier » (site freegan.info, ma traduction).

5 Le freeganisme oppose ainsi au « consumérisme politique » (Dubuisson-Quelliet, 2009, p. 50) une stratégie de « politique préfigurative» (Barnard, 2016, p. 27) (ma traduction): non pas acheter sur le marché des produits plus ou moins "responsables», mais minimiser le recours au marché, qu'il soit du travail ou de la consommation, en construisant d'autres pratiques. Dès le premier usage public du terme "freegan " (Oakes, 2000), celui-ci signifie ainsi «personne vivant gratuitement» par le squat, la minimisation des dépenses, l'autoproduction, le vol, l'auto-stop ou encore la récupération alimentaire. Il est représenté, à partir de 2003, par un groupe d'activistes new-yorkais qui tiennent le site freegan.info, organisent des repas collectifs, des ateliers d'autoréparation et de partage de savoir-faire, du glanage de plantes comestibles en ville, des zones de gratuités ou des séances guidées de récupération alimentaire dans Manhattan (trash tours)... C'est la découverte de ces principes et méthodes, et avec elles de l'ampleur du gaspillage alimentaire, qui me mit sur le chemin de la récupération alimentaire. Issue d'un milieu bourgeois, salariée sur une grande partie de la période, habitant seule sans personne à charge dans une métropole où l'offre économique est dense, j'avais les moyens d'accéder à un mode de vie 
consumériste, c'est à dire d'acheter à neuf, sur le marché formel, ce que j'aurais voulu consommer, modèle qui correspondait de fait à mon éducation et mes habitudes matérielles. Les abandonner releva ainsi d'une décision, et d'une lente rééducation par la pratique, qui fut en grande partie solitaire.

En effet, le freeganisme s'est développé autour d'activités collectives, formatrices d'un groupe, qui ont une " vocation démonstrative » (Dubuisson-Quellier, 2009, p. 106) (elles mettent à l'épreuve par le fait la viabilité sociale des solutions proposées), et ne se limitent pas à l'alimentaire. Cependant, la pratique des trash tours généra une forte médiatisation du groupe, qui devint bientôt un mouvement international fortement centré sur la récupération alimentaire. En France, les deux semblent synonymes: en 2006 est créé le site Freegan Station, qui réunit informations pratiques, textes et forum de discussion. S'il déclare vouloir élargir le mouvement américain, son fondateur met en avant le "régime déchétarien", résumant «en un mot» le freeganisme à « récupérer avant que ce soit brûlé » (Dindar, 2007). Si des initiatives de lutte contre le gaspillage alimentaire apparaissent dans les années 2010, avec des associations nationales (Disco Soupe - illustration 1-, Gars'Pilleurs) et locales (La Maison des Associations), des événements (le Banquet des 5000) et même un restaurant à Paris (Freegan Pony ${ }^{2}$ ), la préparation, distribution ou dégustation d'invendus y servent avant tout de dispositif de sensibilisation. Parfois issus de dons et non de récupération, ces invendus rendent visible et mettent en débat le gaspillage alimentaire sans forcément promouvoir la récupération alimentaire comme mode de vie. Quant aux personnes qui ont, elles, choisi ce mode de vie, si elles échangent informations et photographies sur des sites, forums ou réseaux sociaux (illustration 1), elles n'organisent pas d'évènements publics et semblent s'en tenir à des actions individuelles et affinitaires. En France, activisme politique et mode de vie freegan ne vont donc pas toujours de pair.

7 Une indépendance que l'agenda politique des années 2010, dans lequel entre le gaspillage alimentaire, tend paradoxalement à renforcer. En 2013 est voté le «Pacte national de lutte contre le gaspillage alimentaire ", et 2014 est déclarée "Année européenne de lutte contre le gaspillage alimentaire ». En 2016, la loi Garot oblige les grandes surfaces françaises de plus de $400 \mathrm{~m}^{2}$ à mettre en place des conventions de dons avec des associations. Sur la période, la couverture médiatique (illustration 1) et les dispositifs de sensibilisation croissent, de même que les initiatives économiques redirigeant les restes vers de nouveaux circuits de marchandisation et/ou de défiscalisation, sans remettre en cause le modèle productiviste qui génère ces invendus (Mourad, 2018). Cette constitution du gaspillage alimentaire en problème national repose ainsi, selon Armèle Cloteau et Marie Mourad, sur une prise en charge technicienne et apparemment consensuelle du problème, qui tend à traiter ce problème comme une "cause sans adversaire» (Cloteau et Mourad, 2016). Celui-ci est aussi bien combattu par le plaidoyer institutionnel (la pétition d'Arash Derambarsh appelant la France à promouvoir la lutte contre le gaspillage alimentaire au niveau européen passe le million de signatures début 2018) que géré par des initiatives entrepreneuriales (les applications ZéroGâchis, illustration 1, ou ToGoodToGo), qui réintroduisent des formes d'activisme consumériste peu cohérentes avec l'anticapitalisme et l'anarchisme promu par les premiers freegans. 


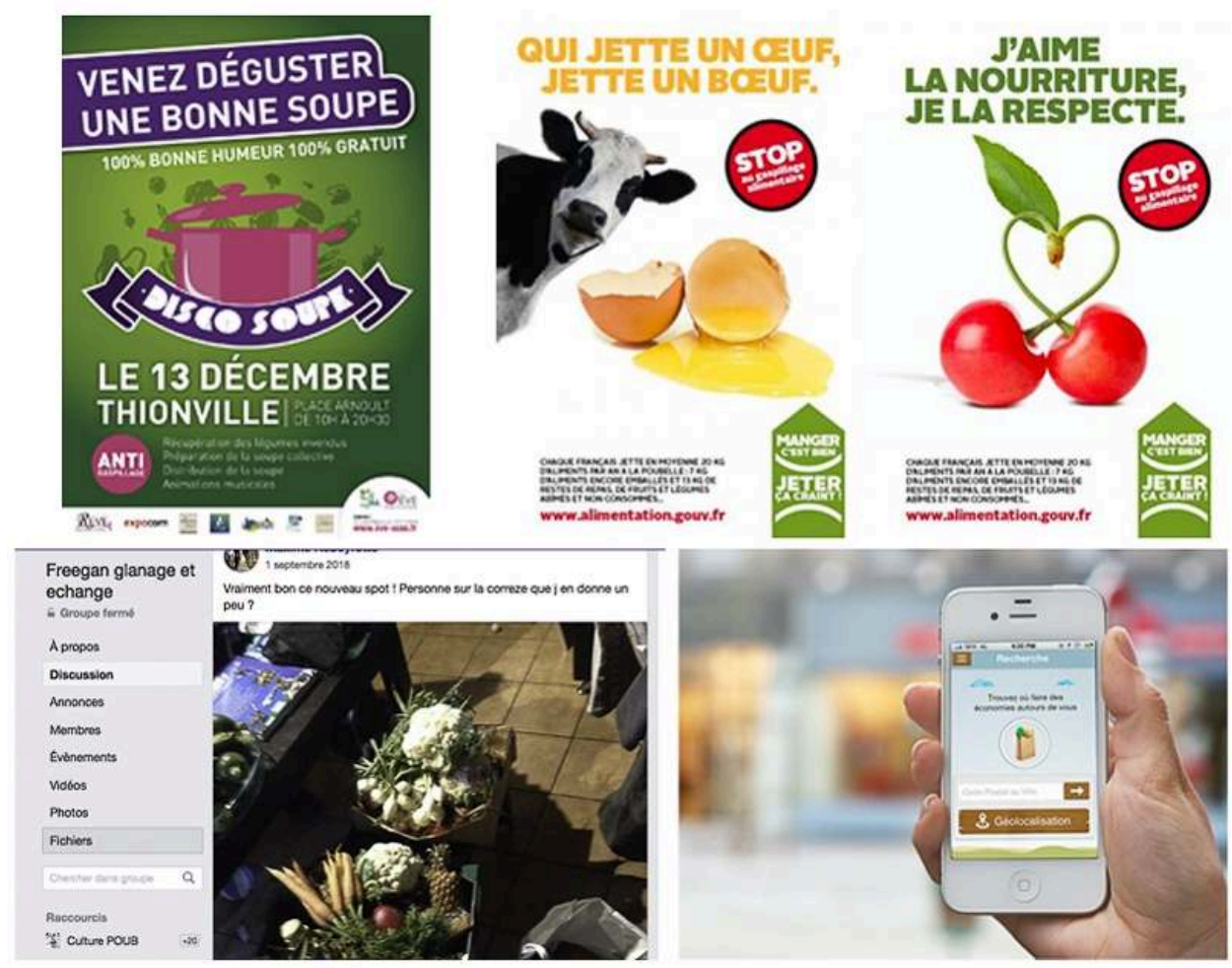

En haut, gauche : affiche de l'association Disco Soupe (@Disco Soupe, 2014).

En haut droite : campagne gouvernementale (2016).

En bas gauche : publication sur le groupe Facebook Freegan glanage et échange (2018)

En bas droite : illustration du site de ZéroGâchis (2018).

8 Tel est le contexte dans lequel je développais ma pratique du freeganisme : constatant qu'il n'existait pas d'événement comme les trash tours (illustration 2), je suivis les conseils d'internautes de Freegan Station et Freegan, glanage et échange, et consultais les fiches horaires des services de propreté de la Ville de Paris pour connaître les horaires de sortie des poubelles de mon quartier. Habitant alors dans le XIX ${ }^{e}$, je fouillais les poubelles d'un Franprix sur la plage horaire indiquée et récupérais en effet des invendus alimentaires en quantités suffisantes pour me nourrir, et même en redistribuer. Dès lors, la récupération alimentaire dans les moyennes surfaces, boulangeries, primeurs, marchés parisiens devint mon mode d'alimentation principale. Je pris rapidement part aux échanges médiatiques autour de cette activité, en m'adonnant à la publication de photographies de récupérations sur Freegan, glanage et échange ; en créant ma propre page, Culture POUB (illustration 2) ; en participant à des Disco Soupe (illustration 2), des récupérations ou distributions des Gars'Pilleurs, des marchés gratuits de La Maison des Solidarités. Dès 2012, je fis l'objet de sollicitations de la part de journalistes, documentaristes, lycéennes, étudiants en journalisme ou en anthropologie filmique, pour témoigner dans des films documentaires (Raimbault, 2012 ; Doumic et Zelez, 2015; Ramirez, 2015), mémoires, TPE ou encore dans des événements consacrés au gaspillage, tels que des projections-débat, y compris pour un film dans lequel je n'apparais pas (Seifert, 2010). J'allais moi-même, en 2016, à la rencontre de freegans new-yorkais, en participant à deux trash tours; j'en documentais un et interviewais l'une des fondatrices du groupe. 
Illustration 2 - Récupération seule ou à plusieurs, informelle ou institutionnelle
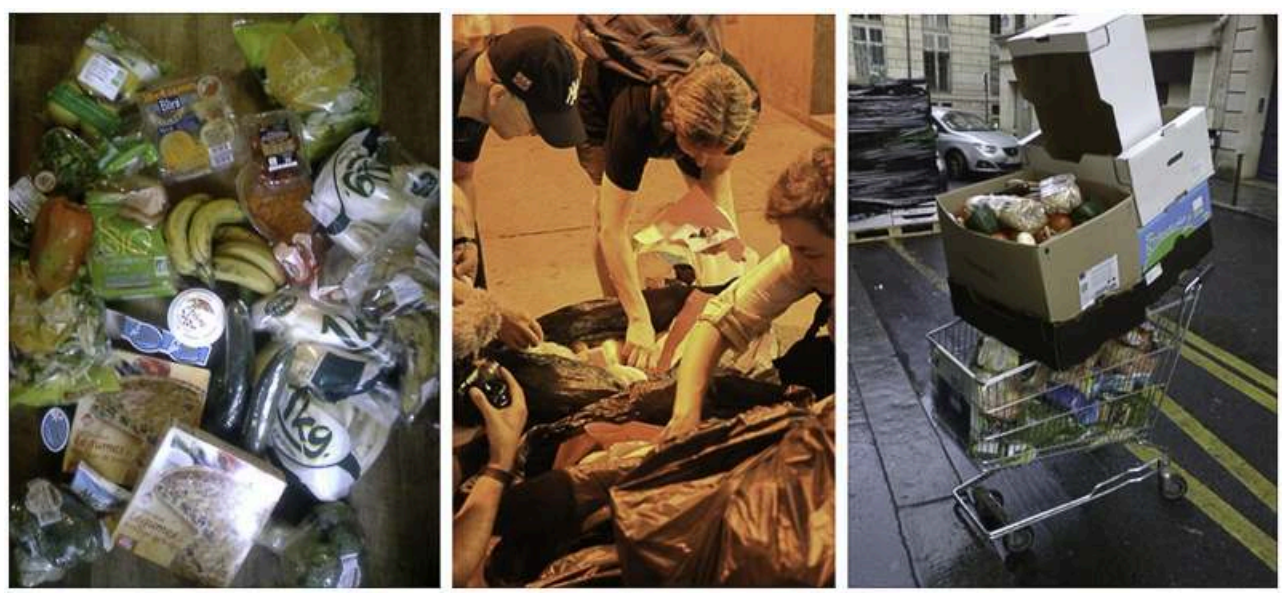

A gauche : exemple de photographie de denrées récupérée publiée sur Culture POUB. Auteur :

J. Guien, Paris XIXe, 2015.

Au centre : participation à un trash tour avec les freegans new-yorkaises. Auteur: J. Guien, Manhattan, 2016.

A droite : réception d'invendus de Monoprix pour une Disco Soupe lors d'un événement du collectif

Place à la Solidarité. Auteur : J. Guien, Paris Ve, 2018.

Ainsi, ma pratique du freeganisme est représentative du contexte français et de ses limites: c'est une pratique individuelle choisie de récupération alimentaire, dont la politisation peine à dépasser le niveau des échanges médiatiques ou des évènements de sensibilisation et de solidarité ponctuels.

\section{Prendre, laisser, partager ? Échanges entre récupérateurs}

Les récupérateurs constituent une première catégorie d'acteurs avec laquelle la récupération occasionne l'échange. Ces interactions me posèrent d'emblée un problème, lié au risque de concurrence que je représentais : si, fouillant une poubelle, je constatais que d'autres personnes y venaient fouiller ou attendaient mon départ pour la fouiller, je risquais d'accaparer leurs ressources et de renforcer leurs difficultés. Le fait de disposer de solutions économiques alternatives désignait mon geste comme accaparement illégitime. Carolsfeld et Erikson (2013) notent ainsi qu'à Vancouver, la récupération alimentaire pouvant être un «dernier recours " mais aussi résulter d'un " choix conscient ", il est nécessaire de respecter une règle informelle, une " étiquette de la poubelle » qui associe la récupération au besoin (ma traduction): Need, not greed ${ }^{3}$. Ainsi dus-je me créer une "étiquette de la poubelle » : ne partir en récupération que cinq minutes avant le passage du camion-benne, afin de passer la dernière. Les longues plages horaires indiquées sur le site de la mairie de Paris donnent en effet l'horaire de sortie des poubelles (par exemple de $5 \mathrm{~h} 30$ à $10 \mathrm{~h}$ ), et non du passage effectif du camionbenne, qui dépend du trajet choisi par les agents de collecte. En connaitre l'heure implique donc de l'observation, et une certaine capacité à ajuster ses pratiques à la situation : l'heure de passage étant soumise aux aléas de la circulation ou du climat, il faut observer à plusieurs reprises son horaire habituel, et toujours s'adapter; ce qui occasionne des échecs (le camion est déjà passé), des accélérations (courir pour parvenir à la poubelle avant les éboueurs) ou décélérations (attendre que le camion se 
montre), des abandons (voyant que quelqu'un fouille déjà la poubelle, et que le camion approche).

Le choix de cette règle fut renforcé par le constat que je fis rapidement de la difficulté concrète de définir une " étiquette » commune. En effet, outre la fréquente barrière de la langue et l'irrégularité de la présence des unes et des autres, la communication s'établit difficilement entre récupérateurs. La récupération alimentaire, dans le cadre parisien où les poubelles sont situées sur le trottoir à des heures diurnes, est une de ces situations de proximité contrainte où les interactions sont minimisées, occasionnant des gestes rapides et de faibles prises de contact. On pourrait l'expliquer par la tension créée par l'activité elle-même, ses exigences physiques et psychiques: surveiller l'imminence de la venue du camion, l'éventuelle hostilité du commerçant, les gestes des autres... Mais aussi, par les effets symboliques de la récupération : le «stigmate » lié à la proximité publique avec les déchets, la « jonction entre la matière et le travailleur » qui s'y opère (Corteel et Le Lay, 2011, p. 25) limite l'identification à cette activité, d'autant plus lorsqu'il s'agit d'un recours au déchet comme ressource directe, et non d'un travail salarié. En six ans, je n'entendis qu'un seul récupérateur se revendiquer « freegan ».

Une autre explication mettrait en cause le risque de relations agonistiques engagé par le partage d'une ressource gratuite dans un contexte non marchand (Mauss, 1969 et 2007 ; Lefort, 1951; Bourdieu, 1976). En effet, je constatais que les personnes à qui j'avais proposé de partager ce que j'avais déjà extrait adoptaient une posture de retrait, cherchant plutôt des prétextes pour n'y rien prélever : «C'est bon, c'est bon. Je regarde juste.»; "Non, non, allez-y, vous êtes jeune, vous.». Cette dernière remarque m'étonna, venant d'une personne beaucoup plus âgée et par là même susceptible, à mon sens, d'éprouver davantage de difficultés que moi à subvenir à ses besoins par une activité aussi physique que la récupération de rue. Il semblait pourtant, de son côté, que c'était mon âge (alors environ 26 ans) qui devait être interprété comme critère de discrimination positive: étant plus jeune, j'avais plus «besoin». Ces concessions m'apparurent comme autant de refus de la position de donataire que j'eus d'abord la naïveté de proposer : en offrant de partager ce que je ne souhaitais pas accaparer, je contestais la relation d'égalité établie de fait par le recours à de semblables ressources. Considérant, du fait de mon âge et de ma classe, que j'aurais dû redistribuer ce que je trouvais, je fis l'erreur de proposer la place inconfortable de donataire à des personnes qui ne la demandaient nullement, mais trouvaient justement dans la récupération un accès indépendant à des ressources sans propriétaire, un moyen de s'en sortir par soimême (voir infra). Devant les quiproquos et les échecs occasionnés par ces propositions, je fus convaincue de la nécessité de pratiquer cette opération le plus tard possible.

13 Je parvins cependant à créer deux relations de partage au cours de mes tournées de récupération. La première, dans le quartier de Crimée, avec un homme d'environ cinquante ans qui, s'il refusa de partager, m'en donna la raison : il ne cherchait que des fromages à pâte dure, du fait de restrictions alimentaires et goûts personnels précis. Dès lors, ce fut comme un jeu de lui mettre de côté les fromages à pâte dure, qu'il acceptait. Ces premiers éléments d'interconnaissance furent suivies de conversations plus variées : j'appris qu'il avait été biffin ${ }^{4}$ et louait un local dans lequel il stockait encore des objets de récupération (alors même qu'il n'avait pas de logement), qu'il ne savait comment vendre. Alors membre d'une association organisatrice de marchés biffins (Amelior ${ }^{5}$ ), je lui proposais de s'y inscrire; ce qu'il fit rapidement, vendant sur le marché et devenant un temps membre actif de l'association. 
Une autre personne avec qui je parvins à nouer des relations sur le tas fut une dame plus âgée qui fouillait les poubelles d'immeubles voisines de celles de la moyenne surface que je fouillais à Belleville. Elle cherchait des objets, aussi la concurrence potentielle n'empêchait-elle pas nos relations. La voyant un jour embarrassée par une trouvaille volumineuse, je l'aidais à l'emporter chez elle. Dès lors, nous entretînmes une communication principalement non verbale, faite de gestes, toujours les mêmes, aux mêmes heures et mêmes endroits: se saluer, se montrer nos trouvailles, s'aider éventuellement à dégager des objets ou à les porter, se souhaiter une bonne journée. Je ne sus pas son prénom; je crus comprendre qu'elle récupérait ces objets pour sa famille, en vue d'un usage direct. La relation, fragile et précautionneuse, se fit autour de l'activité, vecteur d'interactions lorsque les réticences liées au stigmate de la ressource "déchets » sont suspendues. Le paradoxe est que contester ce stigmate en essayant d'établir des relations d'échange fait surgir un autre stigmate, celui du don agonistique et de la dépendance possible à l'aide, sources d'autres réticences à interagir. L'égalité face à la ressource est menacée par la concurrence ou le don, double problème que je choisis d'éviter en passant la dernière.

\section{Prendre, recevoir, demander ? Échanges avec commerçants et employées}

n autre groupe d'acteurs avec lequel la pratique de la récupération met en interaction est le groupe des commerçants ou employées des établissements dont je fais les poubelles. Cette interaction est plus fréquente qu'avec les autres récupérateurs, que j'évite, et plus fréquente qu'avec les passantes, que l'objet de ma pratique n'implique pas directement. En effet, contrairement aux poubelles d'immeubles, où sont mélangés les déchets de divers ménages et qui sont sorties par des gardiens, les commerçants sont responsables du contenu et de la gestion de leur poubelle, qu'ils remplissent et mettent sur le trottoir ; les commerces étant en rez-de-chaussée, vitrés ou ouverts, ils peuvent la voir ; la récupération parisienne ayant lieu dans des espaces publics et non privés (comme les zones de déchèterie encloses des hypermarchés, interdits à Paris) à des heures fréquentées, elle rend possible l'interaction avec les commerçants.

Possible, mais non problématique, croyais-je : les déchets étant res nullius, il n'est pas interdit de les collecter sans rien demander à personne. Je découvris cependant combien il est rare qu'un commerçant demeure neutre ou passif à l'égard d'une pratique qui porte sur ses déchets. Loin d'être traitée comme quelqu'une qui lui fournit un service (tel que la collecte municipale des ordures, fiscalement financée par les ménages et les commerces), la récupératrice est traitée comme quelqu'une qui reçoit un service, ou demande à en recevoir un. Ainsi, les commerçants m'acceptaient ou me refusaient, selon une palette de réactions large : négociation, collaboration, évacuation, et diverses stratégies d'empêchement que je qualifierai de " privatisation des déchets ».

Souvent, la présence régulière sur un lieu de récupération semble n'attirer que peu d'interactions avec les employés; tout au plus me demande-t-on de ne pas gêner les manœuvres de mise au rebut et de ranger derrière moi. La mise au rebut des invendus est un travail (dont se chargent souvent les employés les plus dominés de l'équipe) qui croise celui du récupérateur ; aussi ce dernier est-il fréquemment sommé de veiller à la propreté et l'ordre des poubelles, sous peine de causer une surcharge de travail aux employés ou aux éboueurs, qui d'après les commerçants ne manqueraient pas de le leur 
signaler, voire de leur adresser une "amende ». Le récupérateur est perçu comme source de désordre dans ce monde du déchet, qui n'est pas un chaos mais implique des savoir-faire et des conventions entre travailleurs du déchet ; la récupératrice doit donc être prévenue des règles qui gouvernent cet ordre. Le risque d'amende est souvent utilisé comme prétexte pour éloigner un récupérateur; la nécessité de garder les poubelles propres est très souvent avancée comme condition pour tolérer sa présence. Ainsi, le laisser-faire n'est pas indifférence, mais implique toujours des négociations : on accepte mon geste, si je laisse les lieux dans l'état où je les trouve. Les commerçants se comportent souvent comme s'ils m'octroyaient un droit sur ce dont ils ont précisément dénié toute propriété et dont ils attendent la destruction; signe qu'ils considèrent encore que le déchet est l'objet de leur juridiction.

D'autres commerçants se comportent avec bienveillance. Ils manipulent précautionneusement la poubelle où je fouille; sont polis («Pardon », "attention ») voire amicaux, admettent l'interconnaissance («Bonjour, ça va aujourd'hui?»); facilitent la fouille (en mettant les invendus dans un sac à part, en me disant ce qu'il y a et où); me l'évitent (en m'invitant à me servir parmi des invendus avant qu'ils soient mis à la poubelle, dans un panier, en me passant les rebus de main à main); m'encouragent («Allez-y, on jette plein là-dedans, plein »); parfois, me proposent des denrées dont je ne saurais dire si elles devaient à l'origine être jetées.

Le vaste registre verbal et physique de l'acceptation révèle cependant l'ambiguïté de la relation qu'instaurent ces gestes : la récupératrice prend-elle, la laisse-t-on prendre, ou lui donne-t-on? Certains gestes sont polysémiques : mettre des légumes dans les poubelles que je suis en train de fouiller (surtout si ce ne sont pas des containers, mais des cagettes bien empilées sur le trottoir) est-ce jeter ou donner? Ne rien faire, quand certains me menacent, n'est-ce pas déjà faire quelque chose pour moi ? Une ambiguïté qui peut être levée, comme lorsqu'un commerçant "met de côté " pour un récupérateur, ou le laisse prendre s'il l'aide à ranger (cas fréquent sur les marchés). En ce cas, il y a une forme de contrat, où les termes de l'échange sont explicités. Mais l'ambiguïté peut aussi être entretenue, permettant de faire varier les termes implicites du contrat en demandant un jour de partir, parce qu' " aujourd'hui, y a rien » ou parce que le collectif de travail est indécis à ce sujet. J'entretiens ainsi des rapports instables avec deux primeurs de mon quartier : parfois on me laisse faire, parfois on me passe directement la cagette, parfois on me demande de partir. Car " on" n'est jamais le même : les employés ne sont pas d'accord sur la conduite à tenir. L'un d'eux me dit un jour «Vas-y, prends, lui c'est un chien », désignant son collègue. Un jour où il me demandait au contraire de partir, je lui demandais pourquoi : ma présence lui causaitelle « des problèmes avec son patron? ». Il confirma et je quittais les lieux.

20 S'ouvre ici le vaste registre du refus, allant de l'empêchement des pratiques à l'affrontement physique : admonestations bruyantes (« Ahy ! Ahy ! Allez ! Ho ! »se mit à crier un jour un commerçant, en un cri semblable à celui utilisé par les éleveurs pour faire aller le bétail), interpositions entre la poubelle et moi, atteintes physiques (m'écarter avec le bras, me saisir pour m'empêcher d'avancer, me pousser...), sommations diverses, parfois vigoureuses («Allez, vous dégagez»), parfois gênées (« Faut pas faire ça... S'il vous plaît, mademoiselle, faut pas faire ça. »).

21 Ces réactions sont parfois appuyées sur des références à la Justice: l'amende des éboueurs, les plaintes que je porterais si je m'intoxiquais avec leurs déchets, le fait que ce soit «interdit» de "faire ce que [je] fai[s]», de "toucher à [leurs] effets 
personnels ", à « mon magasin, ma poubelle ». Ces arguments (et surtout, ces pronoms possessifs) montrent l'ambiguïté du statut juridique de la récupération alimentaire, pratique autorisée mais stigmatisée, souvent identifiée au vol ou à d'imprécises pratiques commerciales frauduleuses (voir encadré 1). Le déchet est, juridiquement, abandonné et appropriable par quiconque porte mainmise sur lui; il ne peut, en théorie, devenir "mon déchet", une propriété, sans (re)devenir un bien. Ces observations montrent au contraire que le déchet, qu'il soit issu de biens ou de marchandises, est loin d'être un objet dont tout désir de propriété a été aboli. A la manière du hau ou du mana qui, selon Marcel Mauss, désigne la « force des choses » qui contraint les échangistes à les maintenir en circulation (Mauss, 2007, p. 159), sous peine de malheur, les déchets restent investis de la présence de leur ancien propriétaire, qui vit leur accaparement comme vol, atteinte à son droit voire à sa propre personne.

\section{Encadré 1 - La criminalisation de la récupération en France}

Malgré l'entrée de la récupération alimentaire à l'agenda politique, on observe dans les années 2010 une tendance politique à criminaliser la récupération, aux retombées juridiques variées et ambiguës. Le maire de Nogent-sur-Marne promulgue en 2011 un arrêté interdisant de faire les poubelles, ciblant clairement les récupérateurs professionnels (« les auteurs, partiellement identifiés, sont organisés en réseaux, parfois concurrents, de collectes et de reventes de matériaux et de fripes »), distinguant les « réseaux » du " glanage, notamment en fin de marché ou aux abords des supermarchés et magasins d'alimentation ». Des arrêtés semblables ont été pris à Puteaux, contre la « recrudescence de ressortissants roumains qui venaient récupérer des déchets dans les rues de la ville pour ensuite en faire commerce " (Livois, 2017) ; à La Madeleine, où il est traduit en roumain et bulgare à destination de " personnes qui, certes, sont en souffrance, mais qui parcourent les rues de la ville et ouvrent systématiquement les sacs poubelles » (France-Soir, 2011) ; à Saint-Étienne, interdisant « sans limitation de durée » la « récupération et le chiffonnage » (ibid.). Se défendant de « faire la chasse aux pauvres ", le maire de Nogent rencontra une vive opposition, et son arrêté fut cassé par le Tribunal administratif (TA), de même que celui de Saint-Étienne en 2016. Celui de Puteaux reste en vigueur et a autorisé depuis 2009 environ 150 amendes ; idem pour La Madeleine dont l'arrêté anti-récupération a été validé par le Conseil d'État après saisie par la ligue des Droits de l'Homme.

On observe aussi, sur la période, des plaintes d'enseignes de supermarché pour « vol » à l'encontre de leurs employées pratiquant la récupération. Si leurs procès ont donné lieu à des relaxes, comme à Rouen en 2017, le règlement interne des magasins (Monoprix, Intermarché, Lidl, PSA, Métro...) a permis le licenciement de nombreux employés, dont certains ont ensuite eu recours aux prudhommes.

Ainsi, selon les cas, le référentiel juridique (national ou municipal ; privé ou public), et les démarches (plaintes, amendes, recours aux prudhommes ou au TA) varient. La récupération n'est pas interdite, mais les enseignes et les municipalités ont le droit de promulguer des règlements et des arrêtés qui l'interdisent sur leur espace, dont certains sont ensuite annulés par les prudhommes ou le TA. La récupération est ici aussi placée sous le régime ambigu de la tolérance, laissant le grand public désinformé (ainsi me demande-t-on souvent « Mais... C'est légal ?») 
et les récupérateurs exposés à l'arbitraire des interprétations policières et juridiques. poubelles se trouvant devant les commerces, mon activité est visible et rend visible leur gaspillage; elle rend de plus visible la disponibilité gratuite de leurs denrées. Ces explications maintiennent les commerçants dans leur rôle de commerçant, soucieux de leur réputation et de l'éventuel manque à gagner que je pourrais être. Elles ne rendent cependant pas vraiment compte du caractère particulièrement marqué affectivement de leurs réactions: la récupération génère des conflits au sein du collectif de travail mais aussi au sein même des individus. La violence souvent observée (comme cet épicier qui, devant mon refus de partir, finit par agripper sa poubelle pleine et la rentrer en hurlant dans son magasin) suggère un vécu subjectif ambivalent du gaspillage alimentaire. Sanctionné par une norme sociale très prégnante ( Faut pas gâcher »), alors que le don est, de son côté, valorisé, le gaspillage fait de plus l'objet d'une condamnation officielle croissante. "C'est vrai, ça fait de la peine ", me confia un jour une employée: prise elle aussi dans la contrainte systémique du gaspillage alimentaire, qui intègre le calcul des " pertes » dans celui de la rentabilité, comment pouvait-elle réagir sans ambivalence face au geste qui dévoile ces calculs ? La poubelle est un outil d'évacuation, mais aussi de dissimulation : elle dissimule des rebuts, et le fait même de mettre au rebut. En ce sens, il peut être dangereux de "l'ouvrir », au double sens du terme : de questionner et contester, en fouillant et en faisant émerger ce que l'on enfouit au fond des poubelles.

Aussi le fond des poubelles est-il un lieu très prisé par les commerçants désireux de refuser ma présence sans aller à l'affrontement. S'ouvre ici le vaste domaine des stratégies d'empêchement indirect de la récupération ou "privatisation des déchets ", allant du contrat passé avec une entreprise de collecte privée à la rétention des poubelles dans des espaces de stockage, en passant par la destruction des invendus.

J'observais en effet des absences mystérieuses : certaines enseignes ne mettaient pas ou plus leurs poubelles sur le trottoir à l'heure de passage du camion municipal, faisant appel à des services privés de collecte, leur permettant de passer la poubelle directement aux employés de ces services. Un jour où je tentais de m'interposer, l'un d'eux fut très clair : "on n'a pas le droit». Une autre stratégie, pour les commerces n'ayant peut-être pas les moyens de payer ce service (je n'en observais que pour des grandes chaînes de supermarché), est de retenir ou cacher sa poubelle, comme ce boulanger du XIXe qui, jetant un à trois sacs de farine remplis de pain et viennoiseries par jour, se mit à ne plus sortir sa poubelle qu'à l'arrivée du camion, la passant directement à l'éboueur; ou cette enseigne alimentaire de Belleville dont les invendus n'étaient plus jetés mais stockés dans un caddie laissé jusqu'au passage du camion dans un espace de déchargement privé.

Enfin, la façon de jeter peut empêcher la récupération : les denrées alimentaires sont des biens fragiles qui supportent mal le contact avec les parois du container ou la pression exercée par d'autres déchets. Jeter au fond de la poubelle, déchirer les emballages, écraser et mélanger des aliments peu compatibles, asperger d'eau de javel sont des techniques répandues (quoique l'eau de javel le soit moins que ne le laisse croire l'importante référence qui y est faite dans les médias et discussions), qui permettent un refus indirect et euphémisé de la récupération, malgré la violence des 
pratiques en elles-mêmes (détruire et empoisonner). De même que "laisser prendre " peut être interprété tantôt comme indifférence, tantôt comme bienveillance, " empêcher " peut sembler volontaire ou fortuit. Ce, parce que "mettre au rebut » est une expression vague pour désigner une grande diversité de pratiques, des degrés dans la négation et la destruction. Des déchets abîmés ${ }^{6}$ ou en désordre sont-ils le résultat de pratiques négligentes ou volontaires ? Jette-t-on parce que c'est inutilisable, ou rend-on inutilisable en jetant? Par la réduction en miettes, en bouillie, en poison, l'expropriétaire malheureux de n'avoir pu suffisamment jouir de son bien, ou tirer profit de sa marchandise, semble vouloir s'assurer que personne ne le pourra jamais. Mais peut-on imaginer, à l'inverse, un «bien jeter » qui serait, comme le note Monsaingeon (2017, p. 247) la «forme raffinée de ce processus d'abandon, [...] une réponse moralement acceptable pour le sujet désencombré » ? Jeter, c'est toujours déjà détruire, condamner. Le surcroît de violence destiné à empêcher la récupération montre la contrainte qu'exerce sur les sujets l'injonction marchande à la rotation des produits, à l'abondance et à la « fraîcheur », malgré la condamnation morale du gaspillage.

Illustration 3 - Diversité des techniques de privatisation et de destruction des déchets
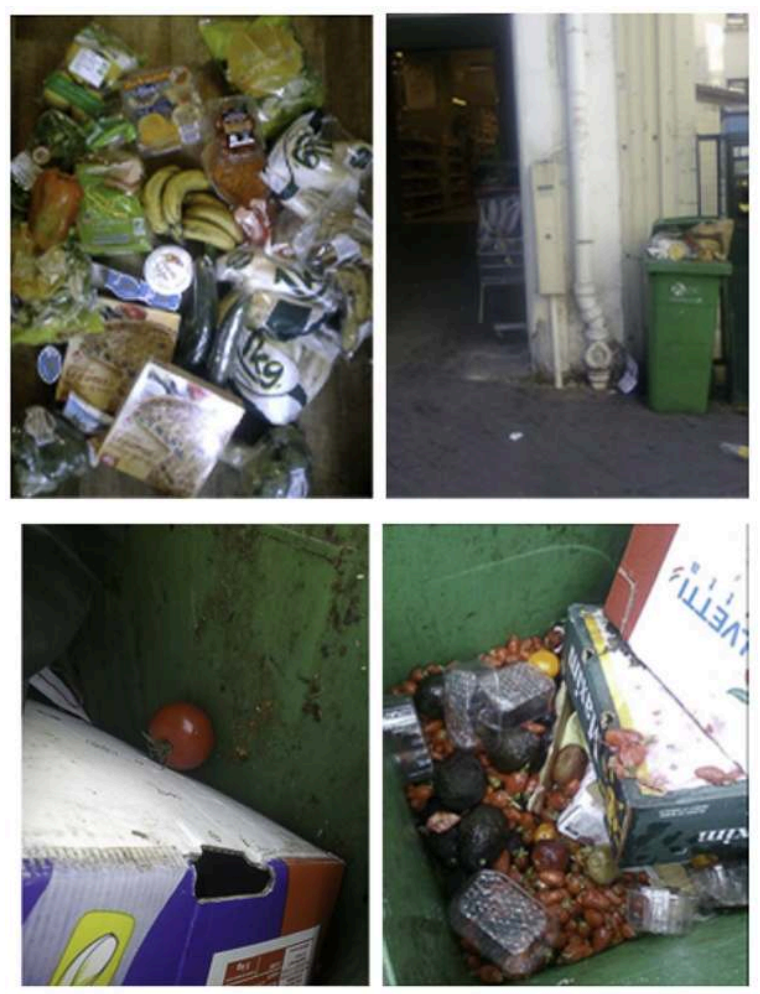

En haut : privatisation des déchets par javellisation (Paris XIXe) et par rétention (Paris XIXe) Auteur : J. Guien, 2013 et 2014

En bas : destructions des invendus (Paris XVIIIe) - Auteur : J. Guien, 2017.

\section{Donner, prendre, protéger ? Échanges avec éboueurs et passantes}

La solidarité témoignée par les éboueurs s'oppose aux démonstrations d'hostilité de certains commerçants, et à l'image menaçante que ces derniers construisent parfois 
d'eux, comme dépositaires d'une vague autorité municipale. Les éboueurs me saluent, se montrent précautionneux au moment d'emporter une poubelle proche de moi ("Attention!», "C'est bon? On peut y aller?»), m'adressent des paroles aimables («Bon appétit!»), somment le boulanger qui leur apportait directement sa poubelle de me laisser fouiller. Je n'eus qu'une seule altercation avec un éboueur, qui me heurta physiquement avec une poubelle pour me signifier que c'était à son tour de s'en occuper. Ce fut l'unique fois où j'eus l'impression de constituer une gêne dans le travail de collecte. Si je veille de fait à leur laisser la place quand le camion parvient à ma hauteur, et à laisser l'espace en ordre, leur arrivée n'est pas un moment de tension.

Les passants entrent aussi en contact ; parfois, en position de don (me proposant une pièce, une denrée...) ; parfois, d'échange verbal, me demandant d'éclairer la situation (« Pourquoi vous faites ça ? Vous n'avez pas de travail ?»). Se pose alors le problème de refuser le don (également proposé par des éboueurs et employées de supermarché) et de justifier de mon activité, délicat dans la mesure où la réponse à cette question n'est pas forcément celle qu'ils attendent, et où je n'ai pas toujours envie de répondre. En effet, je remarquais rapidement que j'étais moi-même en proie à la fermeture relationnelle décrite supra: la récupération implique effort physique (avoir la tête dans la poubelle ; tenir un couvercle, des denrées, porter), attention (surveiller l'avancée du camion, le comportement du commerçant, le passage des voitures), urgence parfois (courir à la poubelle, ramasser le plus vite possible). Je ne suis donc pas toujours disposée pour la conversation, même si elle porte sur les raisons de ma pratique: comment les exprimer rapidement et simplement? Les mots " gaspillage alimentaire " ou "réduction des déchets", devenus communs, suffisent-ils ? À ce problème "pratique » (car lié à la gestion de l'action, notamment du temps) s'ajoute mon refus de bénéficier de ressources dont je n'ai pas un besoin exclusif. Ainsi, je refuse le don et dis en général que je fais cela pour éviter que "ça parte à la poubelle", que "ça soit gâché ». Cela suscite rarement davantage de curiosité, mais achève parfois l'échange dans l'incompréhension: une cliente d'une boulangerie dont je fouillais la poubelle voulu m'offrir un ticket restaurant, que je refusais en lui disant que je préférais récupérer. Elle insista, sur le même registre: "Mais comme ça, vous pourrez acheter !».

Je tente cependant quelquefois d'échanger : si des passants ralentissent, regardent avec stupéfaction les quantités que j'extrais, s'approchent des denrées, je les salue, leur en propose, et parfois ils acceptent, après avoir vérifié que je suis «bien sûre ». Je dois alors tendre les denrées, montrer en même temps la quantité que j'ai à ma disposition, afin qu'eux-mêmes ne craignent pas de me faire concurrence; j'essaie de donner des conseils sur la manière de les consommer ou de récupérer. Les relations sont, là aussi, hésitantes et précautionneuses. Seule une adolescente m'interpella avec enthousiasme sur ma pratique, parce qu'elle avait vu Le scandale du gaspillage alimentaire (Raimbault, 2012) à la télévision et m'avait reconnue; mais son entrain venait plutôt de la découverte que ce film avait été tourné «ici », dans son quartier, avec des denrées récupérées dans la poubelle du magasin qu'elle fréquentait. Je ne me vis attribuer qu'une seule fois le mot «freegan» («vous êtes freegan?») par une passante. Je ne puis témoigner que d'une démonstration directe d'hostilité, de la part d'une troisième. Sa fille d'environ 4 ans, voyant que j'avais posé sur le couvercle de la poubelle des jouets qu'elle contenait, avait tendu la main vers eux. Elle tapa sur sa main et s'écria (en 
me regardant) : «Ça va pas non? Et puis quoi encore ?! ». Faire les poubelles était la dernière des choses qu'elle pouvait lui souhaiter, et cela, il fallait me le faire savoir.

Entre la compassion et l'agression, s'étend enfin le vaste champ de propositions récurrentes que je qualifierais avec Paolo Tabet d'économico-sexuelles, c'est-à-dire d'invitations masculines à l'échange de «services sexuels» contre des " compensations» matérielles (Tabet, 1987). Beaucoup de passants hommes me firent en effet des propositions témoignant de l'existence d'un "continuum» entre les différentes formes d'échanges économico-sexuels, de la prostitution à la dot en passant par les divers cadeaux et invitations (Tabet, 1987). La finalité sexuelle de ces propositions atteint des degrés d'explicitation divers, allant de la proposition de services tarifés à la demande en mariage, en passant par les invitations à déjeuner. Des passants prirent en effet l'alimentaire pour prétexte, me proposant de "m'inviter à déjeuner » ou de " $\left[m^{\prime}\right]$ achete$[r]$ des légumes ", m'appelant "mademoiselle » ou " miss»; un employé de Franprix me demanda mon âge puis m'informa qu'il " cherch[ait] une copine »; un éboueur me proposa de l'épouser ; un passant s'arrêta et me demanda si j'étais "toute seule»; alors que je tentais de faire échouer la conversation en répondant "Là ? En ce moment ? Oui, tu vois bien ", il me demanda si je ne «cherch[ais] pas un protecteur». Un seul (passant) alla à la contractualisation explicite, précisant le tarif et la prestation recherchée.

Les autres propositions (et tout particulièrement ce terme de "protecteur »), dont on peut remarquer qu'elles viennent de tous les groupes d'acteurs étudiés, ne sont pas explicites. En cela, elles sont riches d'enseignement tant sur la perception sociale de la récupération que sur les rapports de genre. Elles témoignent du «lien organique blindé, on dirait, entre la gestion de la sexualité, la division sexuelle du travail et l'accès inégal aux ressources » (Tabet, 2014). D'une part, elle montre l'idée que ces hommes se faisaient de mes besoins au vu de mes pratiques de récupération; d'autre part, l'idée qu'ils se faisaient de l'intérêt que, en tant que femme, j'aurais dû trouver à accepter un statut de dominée. Avisant une situation qu'ils percevaient comme dégradante ou de dernier recours, ces hommes me proposèrent ce que, selon eux, j'étais censée rechercher : un statut économique et social sécurisé en échange de services sexuels. On retrouve ici l'association entre «boulot sale » (lié au déchet) et « sale boulot » (Hughes, 1996 ; Lhuillier, 2005), d'autant plus dégradant lorsqu'il est féminisé (Benelli, 2011, p. 94-103).

31 Outre le biais du genre, jouaient ici ceux de l'âge et de la classe. Comme le dit une employée de supermarché, il était étonnant que fasse les poubelles une fille "jeune et fraîche ». Autrement dit, la jeunesse (alors environ 25 ans) et l'état de mon corps suggéraient que je pouvais accéder à d'autres ressources, en travaillant (car j'étais physiquement valide), en évitant certaines dépenses auxquelles je semblais avoir accès (hygiène et habillement), ou encore en trouvant un ou plusieurs hommes avec qui entretenir des formes d'échanges économico-sexuels. Mon corps faisait ainsi converger un faisceau de représentations et d'attentes concernant le travail des femmes, leur sexualité et leur occupation de l'espace. Souvent penché ou accroupi sur le trottoir, dans une proximité physique forte avec des matières taboues (le bitume foulé par les passants, l'eau charriée par le caniveau), ce corps apparaissait comme «déplacé », sur le mode du déplacement constitutif du tabou de la «souillure " étudié par Mary Douglas (2001) : il n'est pas à la place qui lui revient dans l'ordre social. Comment la jeunesse et la fraîcheur pouvaient-elles s'approcher, et même s'alimenter, s'incorporer, 
des substances périmées, «dépassées "? Comment une femme pouvait-elle s'exposer, seule et dans la rue, à des tâches fatigantes et stigmatisantes? Genré et âgé, ce corps déplacé faisait émerger, en guise de critères de classement, de re-placement, des préjugés concernant la place de la femme dans l'espace (privé, intérieur) et dans la division du travail (domestique, mais aussi sexuel).

\section{Conclusion}

Dans l'ensemble, ces expériences témoignent de la faible identification de la pratique $\mathrm{du}$ freeganisme et du mépris social de la récupération, vue comme échange compromettant avec des matières taboues et des lieux inhabitables : la récupération de rue est toujours conçue comme dernier recours, dont il faudrait émanciper à tout prix les acteurs, fût-ce par la domination, et fût-ce malgré eux. C'est pourtant un aspect fréquemment souligné par les récupérateurs, et particulièrement les récupératrices, que l'indépendance de cette activité, qui en fait un métier digne; un métier, sinon choisi, en tout cas apprécié, car conçu comme une alternative aux «gestions par le haut» des "patrons, qui nous méprisaient, [d]es institutions, qui nous violentaient, [d]es maris» (Collectif Rues Marchandes, 2016). Revendications qui invitent à complexifier la distinction initialement convoquée entre récupérateurs par choix et récupérateurs par nécessité, fondée sur des préjugés de classe qui invisibilisent les luttes populaires (Guien et Ramirez, 2017), et sur des préjugés de genre qui associent systématiquement travail féminin et tâches domestiques, condition féminine et statut de dominée. Rappeler le discours de l'indépendance économique et sexuelle qui soustend les pratiques économiques informelles est à ce titre crucial ${ }^{7}$.

Aidée ou chassée, intimidée ou mise sous tutelle, la récupératrice alimentaire de rue n'est guère laissée à la solitude de son geste. Paradoxalement, c'est dans l'interaction avec ceux qui considèrent aussi le déchet comme une ressource que l'échange est le plus rare, ne s'établissant que si toute menace de concurrence ou de relation agonistique a été suspendue. À l'inverse, ceux qui créent le déchet comme tel, abandonnent, détruisent ou attendent la destruction de denrées comestibles ont tendance à considérer la récupératrice comme parti prenante d'un échange dont ils fixent eux-mêmes les termes, et de façon ambiguë. En effet, outre la diversité et l'instabilité des pratiques de mise au rebut, on constate que beaucoup de gestes sont polysémiques. Laisser prendre, donner, empêcher, prévenir, sont des gestes aussi complexes que ceux de demander, prendre, partager, ou encore accepter de recevoir. Car ces gestes ne sont jamais unilatéraux : parce qu'il n'y a pas de déchet ultime, et que toute chose peut acquérir, perdre ou reprendre de la valeur, le jeteur n'est pas indifférent à la récupération, toujours partiellement vécue comme un échange, refusé, concédé ou négocié. Dans ce contexte, et vue la tendance à la criminalisation de la récupération, cette pratique est souvent placée sous le régime ambigu de la tolérance, exposant les récupérateurs à l'arbitraire d'interprétations variables et de requêtes contradictoires. 


\section{BIBLIOGRAPHIE}

Barles S., 2005. L'invention des déchets urbains 1790-1970. Ceyzérieu dans l'Ain, Champ Vallon, 297 p.

Barnard A.V., 2016. Freegans. Diving into the wealth of food waste in America. Minneapolis, University of Minnesota Press, 294 p.

Bazin H., Rullac S., 2011. Étude qualitative portant sur les conditions de vie des biffins en Île de France. Région Ile-de-France. Association Aurore/Unité Société de la Direction du Développement Social, de la Santé et de la Démocratie régionale de la Région Ile de France, 106 p.

Benelli N., 2011. Nettoyeuse. Comment tenir le coup dans un sale boulot. Paris, Séismo, 218 p.

Benelli N., Corteel D., Debary O., Florin B., Le Lay S., Retif S., RETIF, 2017. Que faire des restes ? Le réemploi dans les sociétés d'accumulation. Paris, Presses de la fondation nationale des sciences politiques, $111 \mathrm{p}$.

Bertolini G., 1978. Rebuts ou ressources ? La socio-économie du déchet. Paris, éd. Entente, 270 p.

Bourdieu P., 1976. Les modes de domination. Actes de la recherche en sciences sociales vol. 2, $\mathrm{n}^{\circ} 2-3$, p. 122- 132 .

Carolsfeld A. L., Erikson S. L., 2013. Beyond Desperation: Motivations for Dumpster ${ }^{\mathrm{TM}}$ Diving for Food in Vancouver. Food and Foodways, vol. 21, n 4, p. 245-266.

Cirelli C., Florin B., (dir.), 2015. Sociétés urbaines et déchets. Eclairages internationaux. Tours, Presses Universitaires François-Rabelais, 450 p.

Chouatra P., Grimaldi Y., 2014. «De seconde main ». Vendeurs de rue et travailleurs sociaux face à face dans la crise. Paris, L'Harmattan, 249 p.

Cloteau A., Mourad M., 2016. Action publique et fabrique du consensus. La « lutte contre le gaspillage alimentaire » en France et aux États-Unis. Gouvernement et action publique, vol. $1, \mathrm{n}^{\circ} 1$, p. 63-90.

Collectif de recherche-action RUES MARCHANDES, 2016. Les biffins récupérateurs vendeurs, guide indigène de la ville [En ligne]. http://recherche-action.fr/ruesmarchandes/download/ etude_sur_les_biffins_en_ile_de_france/Guide-biffins-edition-A4.pdf Corteel D., Le Lay S., (dir.), 2011. Les travailleurs des déchets. Toulouse, ERES, 2011, 336 p.

Culture POUB. Page Facebook. https://www.facebook.com/culturepoub/ (dernière consultation : mai 2018)

Dindar J., 2007. Poubelle la vie. Émission 66 minutes. M6, $12 \mathrm{mn}$.

Douglas M., 2001 [1 1 ère ed. : 1971]. De la souillure. Essai sur la notion de pollution et de tabou. Paris, La Découverte, $205 \mathrm{p}$.

Doumic L., Zelez E., 2015. Les tribus de la récup. Documentaire, THM, $52 \mathrm{mn}$.

Dubuisson-Quellier S., 2009. La consommation engagée. Paris, Presses de la fondation nationale des sciences politiques, $143 \mathrm{p}$.

Ellis C., Adams T. E., Bochner A. P., 2010. Autoethnography: An Overview. Forum Qualitative Social Research [En ligne], vol. 12, n 1, Art. 10, 2010. http://www.qualitative-research.net/index.php/ fqs/article/view/1589/3095 
France Soir, 2011. Un maire traduit ses arrêtés anti-mendicité en roumain et en bulgare. FranceSoir, 8 août 2011.

Freegan. Site internet. https://freegan.info/ (dernière consultation : mai 2018).

Freegan, glanage et échange. Groupe Facebook. https://www.facebook.com/groups/ 331265793613006/ (dernière consultation : mai 2018).

Freegan Pony. Site internet. http://freeganpony.com/ (dernière consultation : octobre 2018).

Freegan Station. Site internet. http://freegan.fr/ (dernière consultation : mai 2018).

Guien J., Ramirez V., 2017. Travailler à consommer. Terrains \& travaux, n 31, p. 45-62.

Hughes E. C., 1996. Métiers modestes et professions prétentieuses : l'étude comparative des métiers. In E. C. Hughes, Le regard sociologique. Essais choisis, Paris, EHESS, p. 123-135.

Lefort C. 1951. L'échange ou la lutte des hommes. Les Temps modernes, p. 1401-1417, 1951.

Lhuillier D., 2005. Le « sale boulot ». Travailler, n 14, p. 73-98.

LISRA (Laboratoire d'innovation sociale par la recherche-action), 2017. Qu'est-ce que la recherche-action ? [En ligne]. http://recherche-action.fr/labo-social/2017/08/11/rechercheaction-en-laboratoire-social/ (consulté le 08/04/19)

Livois D., 2017. À Puteaux, fouiller dans les poubelles c'est 33€ d'amende. Le Parisien, 14 février 2017.

Mauss M., 1969. Don, contrat, échange. In Euvres. Paris, Ed. de Minuit, tome III, 734 p.

Mauss M., 2007 [ $1^{\text {ère }}$ ed. : 1925]. Essai sur le don. Paris, PUF, 248 p.

Milliot V., 2012. Les biffins ou l'organisation de la misère ?. Territoires, p. 46-49.

Monsaingeon B., 2017. Homo detritus. Critique de la société du déchet. Paris, Seuil, 279 p.

Mourad M., 2016. Recycling, recovering and preventing "food waste": competing solutions for food systems sustainability in the United States and France. Journal of Cleaner Production, $\mathrm{n}^{\circ} 126$, p. $461-477$.

Mourad M., 2018. La lutte contre le gaspillage alimentaire en France et aux Etats-Unis. Mise en cause, mise en politique et mise en marché des excédents alimentaires. Doctorat, Partis, Institut d'études politiques, $551 \mathrm{p}$.

Oakes W., 2000. Why freegan? Tract auto-produit.

Raimbault M.-P., 2012. Le scandale du gaspillage alimentaire. Documentaire Arte, $50 \mathrm{mn}$.

Ramirez V., 2016. Les nouveaux modernes. Documentaire, ADEME,45 mn,.

Seifert J., 2010. Dive! Living off America's waste. Documentaire, Seifert, $42 \mathrm{mn}$.

Stengers I., 2006. La Vierge et le Neutrino. Paris, Les Empêcheurs de penser en rond, 286 p.

Syndicat du TRavail Sexuel (STRASS), 2014. Charte [En ligne].

Tabet P., 1987. Du don au tarif. Les relations sexuelles impliquant une compensation. Les Temps Modernes, vol. 42, n 490, p. 1-53.

Tabet P., 2014. Échange économico-sexuel et continuum. In Broqua C. et Deschamps C., (dir.), L'échange économico-sexuel. Paris, Ed. de l'EHESS, p. 19- 60.

Weber F., 2009. Le calcul économique ordinaire. In Steiner P. et Vatin F., Traité de sociologie économique. Paris, PUF, p. 367-407. 
Zéro Gâchis. Site internet. https://zero-gachis.com (dernière consultation : octobre 2018).

\section{NOTES}

1. Notre accord des genres suit ici le modèle proposé par Isabelle Stengers dans La vierge et le Neutrino, «à savoir l'utilisation arbitraire du féminin de temps en temps. L'effet de surprise me semble plus adéquat au but recherché que la lourdeur des doublets. » (Stengers, 2006, p. 7, n. 1). Lorsque je me désigne comme récupératrice, cependant, je n'applique pas ce modèle d'utilisation arbitraire, mais renvoie au fait que je suis assignée au genre féminin.

2. Le Freegan Pony, lieu ouvert dans le XIX ${ }^{\mathrm{e}}$ à Paris en 2015, est un restaurant associatif qui collecte des surplus alimentaires à Rungis servis à prix libre le soir ; sur son site, le freeganisme est présenté comme «mode de vie alternatif qui lutte contre le gaspillage alimentaire et la pollution générée par le surplus de consommation »; si «d'autres modes de consommation, de transport, de travail et du logement dans la société occidentale " sont mentionnés, le travail réalisé par les membres du Freegan Pony sur son site (restauration, Ateliers du Bon Goût...) ou sur des évènements éphémères extérieurs (festival WeLoveGreen...), est consacré à l'alimentaire.

3. Slogan que l'on pourrait traduire par : « la nécessité avant l'avidité ».

4. Les biffins ou récupérateurs-revendeurs sont les personnes qui récupèrent pour les revendre les objets et les matériaux abandonnés dans les poubelles et/ou dans la rue. On les appelle parfois chiffonniers, puciers ou encore ferrailleurs. A ce sujet, voir notamment Bazin et Rullac, 2011 ; Milliot, 2012 ; Chouatra et Grimaldi, 2014 ; Guien et Ramirez, 2017.

5. L'association Amelior, fondée en août 2012, a pour but de favoriser la reconnaissance du métier de biffin et l'amélioration de ses conditions d'exercice, notamment par l'organisation de marchés (Doumic et Zelez, 2015). J'ai travaillé bénévolement à cette association de 2012 à 2015 ainsi que quelques semaines en 2017.

6. Dans le registre non alimentaire, il n'est pas rare de trouver dans les poubelles des vêtements présentant tous une semblable entaille faite aux ciseaux.

7. Il pourrait également être heuristique de rapprocher ce discours de celui des militantes du travail du sexe, qui revendiquent le caractère économique et autonome de leur activité. De fait, la plupart des propositions citées, par leur caractère implicite, expriment toute la méconnaissance sociale du travail du sexe, tabou dans sa réalité vécue comme dans sa diversité. Associé au seul «trottoir », il est toujours perçu comme une activité dominée enchâssée dans des relations personnelles, et non comme un «travail sexuel autogéré », possiblement encadré par un «statut professionnel ", exercé dans le cadre de relations contractuelles explicites entre personnes autonomes et protégé par des actions et une solidarité collectives (Strass, 2014).

\section{RÉSUMÉS}

La pratique de la récupération alimentaire en milieu urbain (ou « freeganisme ») est l'occasion d'interactions et d'échanges avec les passants, les commerçants, les travailleurs des déchets ou les récupérateurs. Portant un regard autoethnographique sur six années de pratique régulière de la récupération alimentaire dans les rues parisiennes, l'auteure propose d'étudier ce que ces 
interactions parfois conflictuelles nous disent du statut ambigu du déchet, de la récupération et du corps féminin aujourd'hui.

Urban foraging and dumpster-diving (also known as «freeganism ») in the streets are practices that create various interactions and exchanges with people passing by, working in shops or collecting waste. Through an autoethnographical approach, the author analyses six years of dumpster-diving in the streets of Paris, in order to study what those interactions (and, sometimes, those conflicts) disclose about the ambiguous status of waste, waste recovery and women's body today.

\section{INDEX}

Keywords : dumpster-diving, freegan, trades, street, waste

Mots-clés : récupération alimentaire, freegan, échanges économiques, rue, déchets

Thèmes : Sur le Champ - Sur le Terrain

\section{AUTEUR}

\section{JEANNE GUIEN}

Jeanne Guien, jeanne.guien@gmail.com, est doctorante en philosophie à l'Université Paris 1 Panthéon-Sorbonne, laboratoire du CETCOPRA. Elle a récemment publié :

- Guien J., Vuillermet H. (Textes choisis et présentés par), 2018. La technique. Paris, Flammarion, $240 \mathrm{p}$.

- Guien, J. et Ramirez V., 2018. Travailler à consommer. Terrains \& travaux nº 31, p.45-62

- Guien J., 2016. Ivresse et gueule de bois - un Noël à Marseille. Techniques \& Culture, n 65-66, p. 64-73. 